\title{
Advancements in Dynamic Scene Projection Technologies at the U.S. Army Aviation and Missile Command
}

\author{
D. Brett Beasley and Daniel A. Saylor, \\ Optical Sciences Corporation, \\ www.opticalsciences.com \\ P.O. Box 8291, \\ Huntsville, AL 35808
}

\begin{abstract}
This paper describes the recent addition, characterization, and integration of emerging technologies for dynamic infrared scene projection at the US Army Aviation and Missile Command's (AMCOM) Advanced Simulation Center (ASC). Infrared scene projection performs a vital role in the daily testing of tactical and theatre missile systems within these Hardware-in-theLoop (HWIL) laboratories. Topics covered within this paper include the addition and characterization of new Honeywell and Santa Barbara Infrared emitter arrays, a five-axis flight motion table test configuration, unique calibration/NUC schemes, added software support, verification/validation results, and supplemental projection systems. A new dynamic IR scene projector technology based upon the Digital Micromirror Device ${ }^{\mathrm{TM}}$ is also presented in the paper, as well as example imagery from several of the projector systems.
\end{abstract}

Keywords: Infrared, Scene Projection, Digital Micromirror Device, Simulation, FPA testing, Hardware-in-the-loop.

\subsection{INTRODUCTION}

The Advanced Simulation Center is located within the AMCOM Research, Development and Engineering Center (RDEC). The ASC provides high-fidelity, value-added hardware-in-the-loop simulation support to Program Executive Officers (PEO) and Project Managers (PM) who are responsible for developing and fielding tactical precision guided missiles and submunitions for the U.S. Army. The ASC is also engaged in cooperative HWIL simulation tasks which support other DoD Agencies, NATO members and other U.S. Allies. This test support focuses primarily on testing missiles/munitions employing infrared, millimeter wave, and microwave sensors and seekers. ${ }^{1}$

The ASC has six simulation facilities for testing systems which utilize IR sensors or seekers. The ASC IR HWIL facilities are capable of generating dynamic in-band IR images in real-time using advanced IR projector and scene generation computer equipment. This paper provides an overview of the recent advancements made to the IR projector capabilities resident within the ASC.

\subsection{DEVELOPMENTS IN DYNAMIC PROJECTOR TECHNOLOGIES AT AMCOM}

\subsection{HONEYWELL TECHNOLOGY CENTER (HTC) EMITTER ARRAY BASED PROJECTORS}

The dynamic infrared scene projectors built around the emitter array based technology developed by the Honeywell Technology Center continue to play a significant and vital role in the HWIL test support provided by AMCOM. The previous year's efforts have seen the continued operation, characterization, and pursuit of advancements/modifications to these systems thereby maintaining a leading edge test capability. The following sections discuss many of these advancements, along with results from ongoing operation and characterization efforts.

\subsubsection{Operational Advancements}

\subsubsection{Calibration of the HTC Projector}

Calibration of the IRSP is a critical and fundamental step in the preparation for HWIL testing. While a through discussion of the calibration procedure is beyond the scope of this paper, the application of one particular technique to the calibration of the IRSP is presented. This technique, like many within the laboratory environment, recognizes a specific limitation in the test 
setup and attempts to address this limitation with an acceptable solution. Specifically, the technique reviewed addresses the inability of the laboratory HWIL setup to simulate cold tactical backgrounds.

The scene projector system cannot support all possible temperature regions of interest. In particular, background temperatures observed in captive flight test (CFT) runs averaged $\sim 0^{\circ} \mathrm{C}$ while the projector minimum background temperature was limited to $\sim 15^{\circ} \mathrm{C}$. It was determined that, rather than duplicating absolute temperature levels, the relative energy levels between objects of interest and the background would be simulated. By duplicating the same amounts of 'target energy' above background, the seeker focal plane array would produce the same number of 'delta counts' out. Further reinforcing the legitimacy of this approach for the specific UUT in question is the method of operation of the UUT itself. The system uses a 'floating offset' level which can be preset to an arbitrary value prior to a test run. This allows for the absolute object count levels output by the seeker to vary run-to-run while maintaining constant delta count levels between objects. As such, it is only this delta count value for each object, with respect to the background, which is of importance.

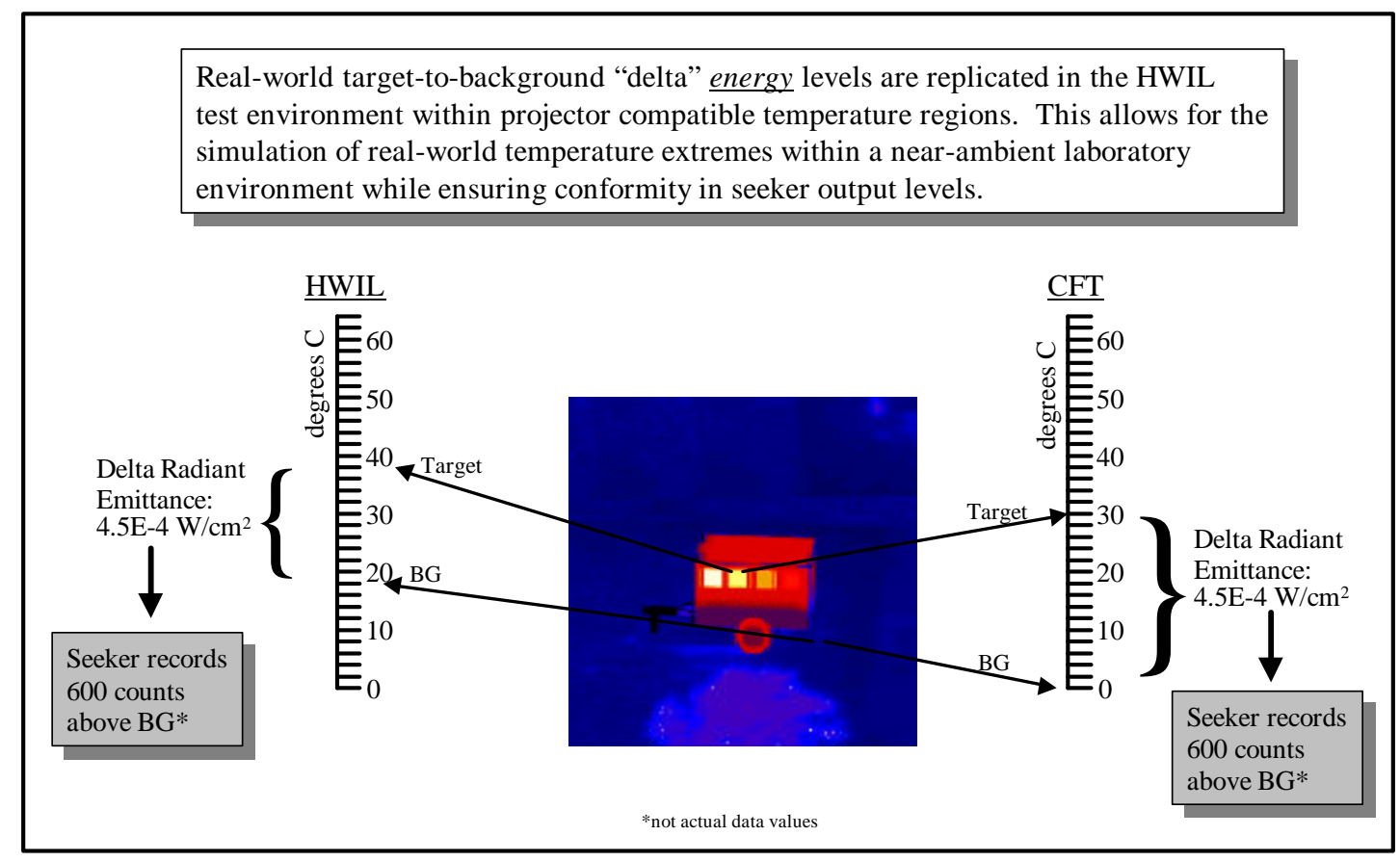

Figure 1 - Delta Radiance Mode

Figure 1 above illustrates this "delta energy" approach. Because the higher laboratory background does fill the detector 'wells' to a greater extent than would be observed in the real-world, this approach is limited to only moderate excursions from the actual background temperatures. Attempting to operate at significantly higher backgrounds than would be observed in real-world scenarios could significantly reduce the dynamic range of the UUT. Validation and verification procedures were performed to insure the replication of the infrared imagery was acceptable. Results from these procedures are discussed below.

\subsubsection{Automated Calibration Software}

Another development in the operational capability within the AMCOM RDEC facilities for calibration of the IRSP is the implementation of an automated closed-loop calibration control panel within the WISP System Control Software (SCS) package. Calibration of the IRSP is performed using the UUT as the data collection device. The relationship between the projector input values and the measured UUT output is automatically mapped over the entire IRSP input range. Knowledge of the UUT's response curve allows for the determination of the required IRSP calibration information. The SCS calibration panel provides optional features for the configuration of such parameters as the number of frames over which to average a particular sample and the background level over which the array should be held during calibration. Graphical display of the measured output is provided as well as storage of all relevant information. Additional capabilities added to the SCS over the previous year are summarized in Table 1. 


\begin{tabular}{|c|c|}
\hline Feature Added & Capability \\
\hline Annealing Panel & $\begin{array}{l}\text { GUI for annealing any sub-region, or all, of the array with user } \\
\text { selected pattern, level, and duration. }\end{array}$ \\
\hline Supervisor Panel & $\begin{array}{l}\text { Password protected GUI allowing for control of sensitive setup } \\
\text { parameters. Configurations stored in Windows Registry. }\end{array}$ \\
\hline Camera Interfaces & $\begin{array}{l}\text { Additional camera interfaces added including Indigo Merlin and } \\
\text { generic "User Defined" camera types. }\end{array}$ \\
\hline Data Analysis & $\begin{array}{l}\text { Additional data analysis features added to "Image Capture" panel } \\
\text { including row and column profile plots and pixel value overlays. }\end{array}$ \\
\hline Snapshot Support & Supports operation of "snapshot" mode. \\
\hline Calibration Panel & $\begin{array}{l}\text { GUI panel to provide user designated background levels during } \\
\text { closed loop autonomous calibration. }\end{array}$ \\
\hline
\end{tabular}

TABLE 1: Projector Software Additions and Upgrades

\subsubsection{Validation and Verification (V\&V) Efforts Using the HTC Projectors}

Central to the application of any projector technology to HWIL testing is the validation and verification of the output imagery. AMCOM performs V\&V activities for each seeker undergoing closed-loop testing. A thorough discussion of the $\mathrm{V} \& \mathrm{~V}$ process implemented at the AMCOM RDEC for IR HWIL simulations is not presented here. The reader is referred to the paper "Captive flight test-based infrared validation of a hardware-in-the-loop simulation" also within this proceeding ${ }^{2}$. Presented here is a brief discussion of the results for a recent validation/verification.

Using the "delta energy" calibration technique described earlier, the projector system was calibrated and operated. Validation data was collected using captured imagery from the CFT, SGI digital input stream, and the HWIL projected output. Figures 2 and 3 illustrate the comparison made between the SGI digital input and the projected output as captured by a surrogate UUT. Of primary interest in this case was the detailed armored vehicle target within the center of the input SGI frame. To increase the sampled resolution over this area of interest, the projector collimator and UUT were set to 'zoom in' on the target. The solid white line across each image illustrates the row of data selected for comparison. Figure 4 compares the slices taken from the imagery in Figures 2 and 3. Very good agreement can be seen across the two images

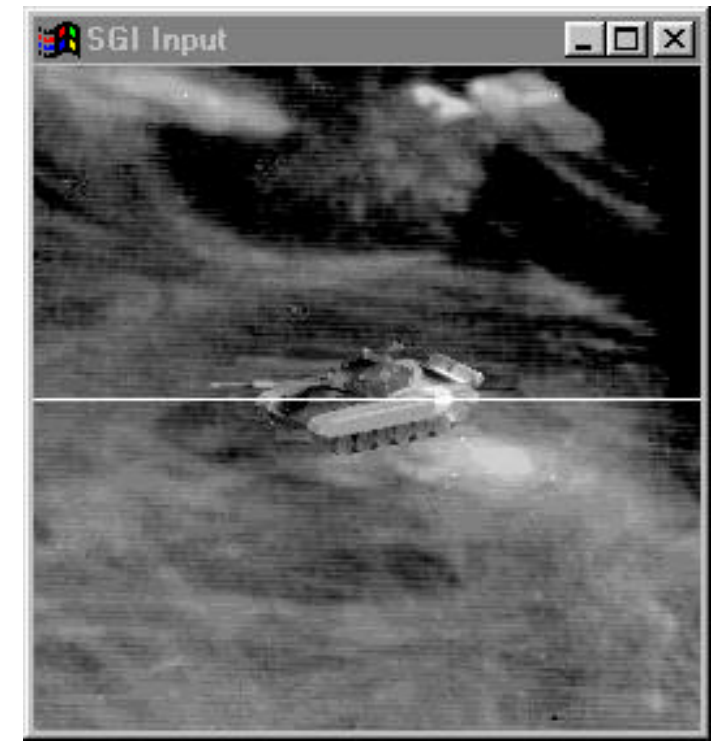

Figure 2 - SGI Digital Input

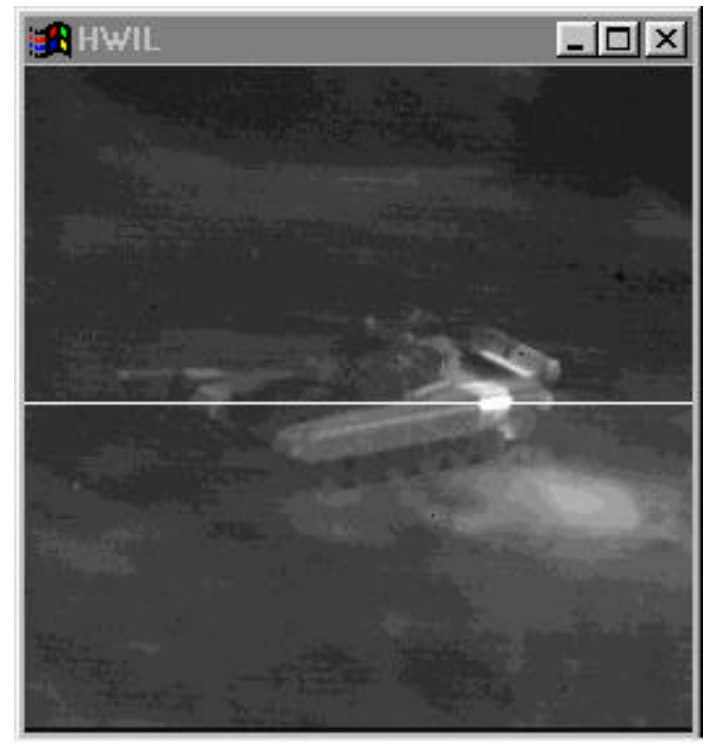

Figure 3 - HWIL Output 


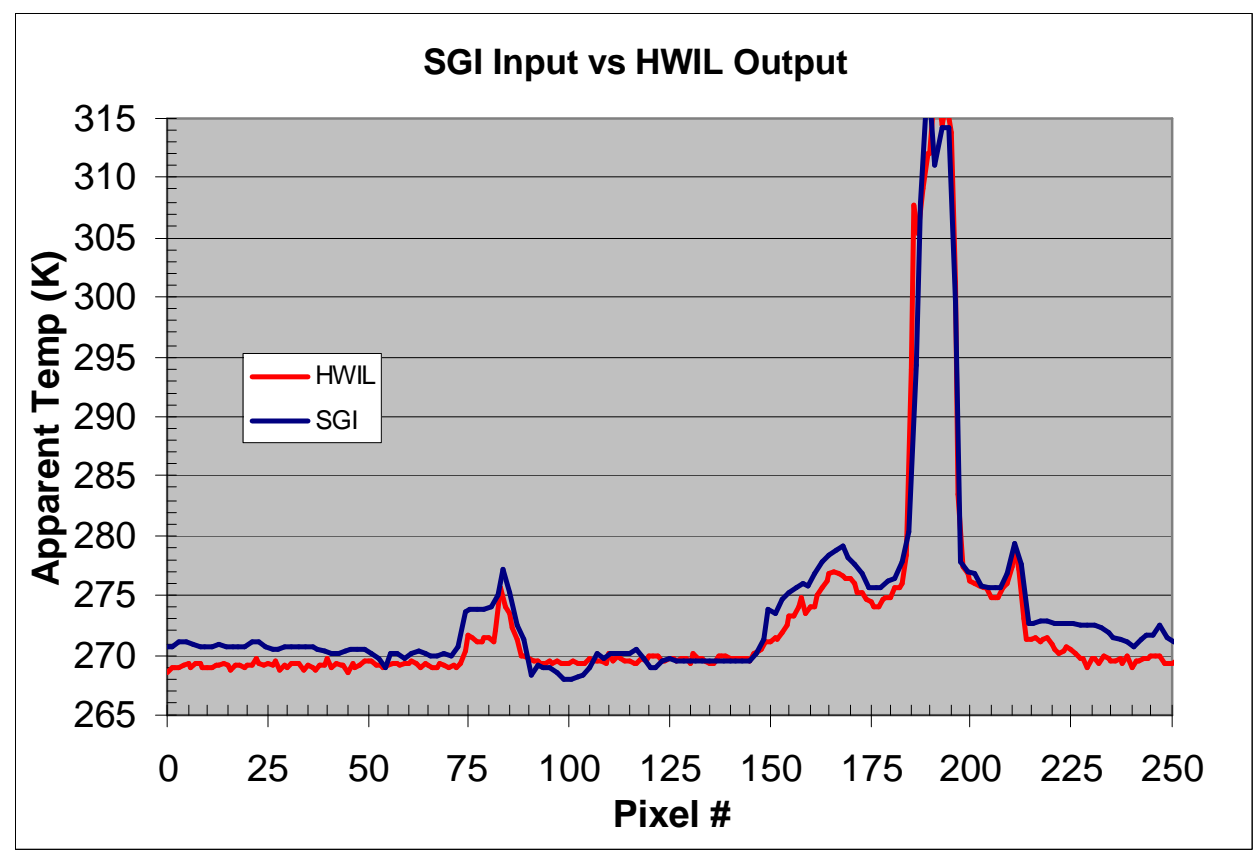

Figure 4 - Comparison of SGI Input and HWIL Output

\subsubsection{System Characterization of the HTC Projector}

AMCOM RDEC personnel, in addition to performing the calibration, verification and validation of the IRSP, continue to investigate and document the performance envelope for each IRSP technology resident with the HWIL laboratories. One area of the Honeywell emitter array performance that AMCOM has recently been investigating is the efficiency in the thermal transfer mechanism and the amount of residual heat remaining in the substrate. It was noted that the measured output of a specific local region within the emitter was influenced by the level to which other large sections of the array were simultaneously driven. Figure 5 shows the average output over a statistically large area on the UUT when illuminated by an $\sim 100 \times 100$ area of the emitter array. The output of the UUT is recorded as the emitter is driven across the entire input range. Additionally, the output for similar measurements is shown where a large array area outside the $100 \times 100$ central region is held at a constant midrange input level. The variation in the measured output due to the inclusion of this background level is also shown in Figure 5. Initial analysis suggests that driving the background emitters creates a low-level residual substrate heating effect which is not completely removed by the thermal control system. Further analysis to determine the extent, if any, to which modification of the coolant flow rate effects this behavior are underway.

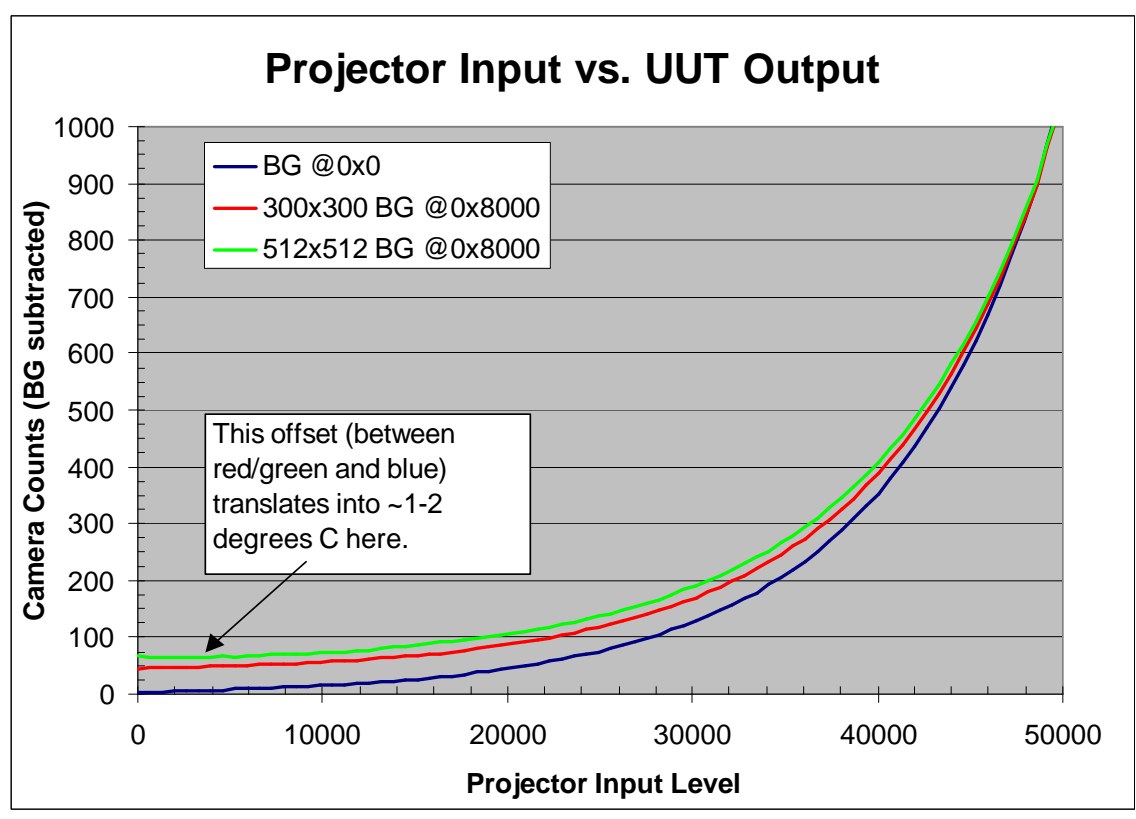

Figure 5 - Residual Thermal Energy Data 


\subsubsection{Hardware Advancements}

\subsubsection{Extended Pupil Collimator}

The testing of each seeker within a HWIL environment brings unique requirements to the test facility. One particular seeker currently under testing at the AMCOM RDEC requires simultaneous dual-band stimulation in RF and IR bands. To address the RF stimulation requirements, the UUT is integrated into a large chamber compatible with RF projection. The introduction of the IR projected imagery required the use of a dichroic beamcombiner and an 'extended pupil' optical collimator $^{3,4}$. This collimator places the exit pupil approximately ten feet from the last optical element within the collimator and provides sufficient relief for introduction of the dichroic and placement of the IRSP and collimator outside the RF chamber. A ray trace of the object plane, collimator optics, dichroic, and exit pupil along with a list of specifications are shown in Figure 6.

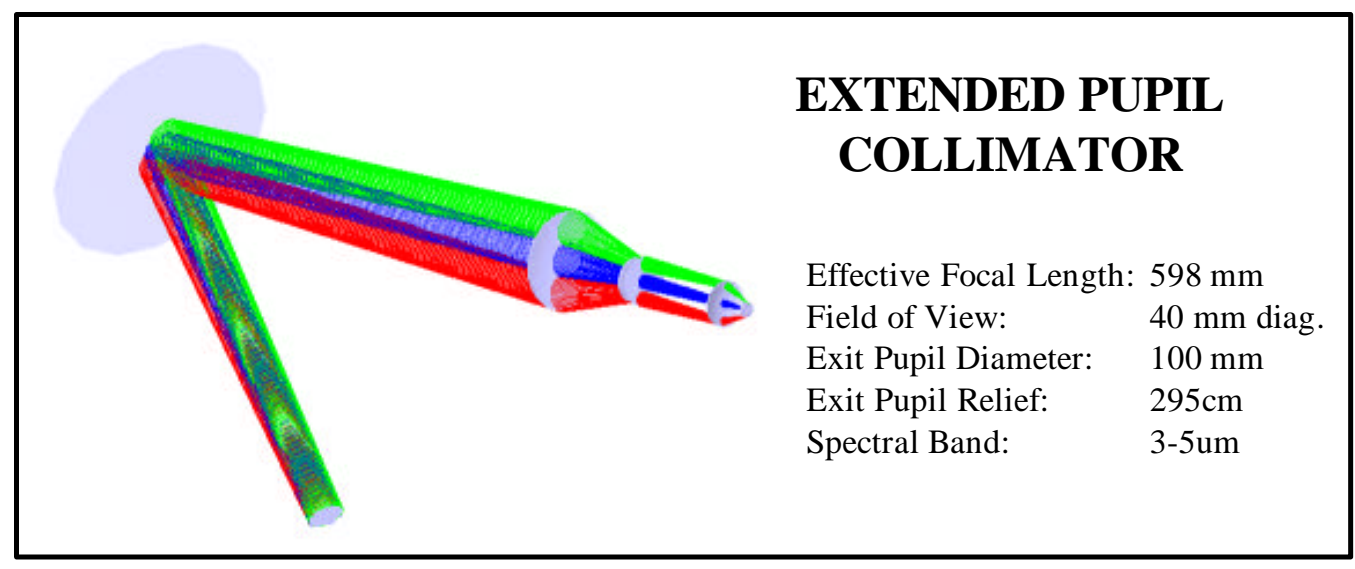

Figure 6 - Extended Pupil Collimator for Dual-Mode HWIL Application

\subsubsection{FMS Mounting of the HTC Projector}

True line-of-sight (LOS) simulation requires both the placement of the UUT into a 3-axis flight motion simulator and placement of the projector device onto an outer two-axis gimbal. AMCOM RDEC has performed preliminary integration of the Honeywell emitter based IR scene projector system onto the outer axis of the 5-axis FMS for closedloop HWIL testing. Figure 7 shows a picture of the system as configured for preliminary checkout.

To support the use of the IR scene projector on the FMS, an FMS-compatible MWIR zoom collimator has been designed and will shortly be delivered for integration into the AMCOM RDEC facility. Figure 8 below lists several system specifications and shows a raytrace for this collimator.

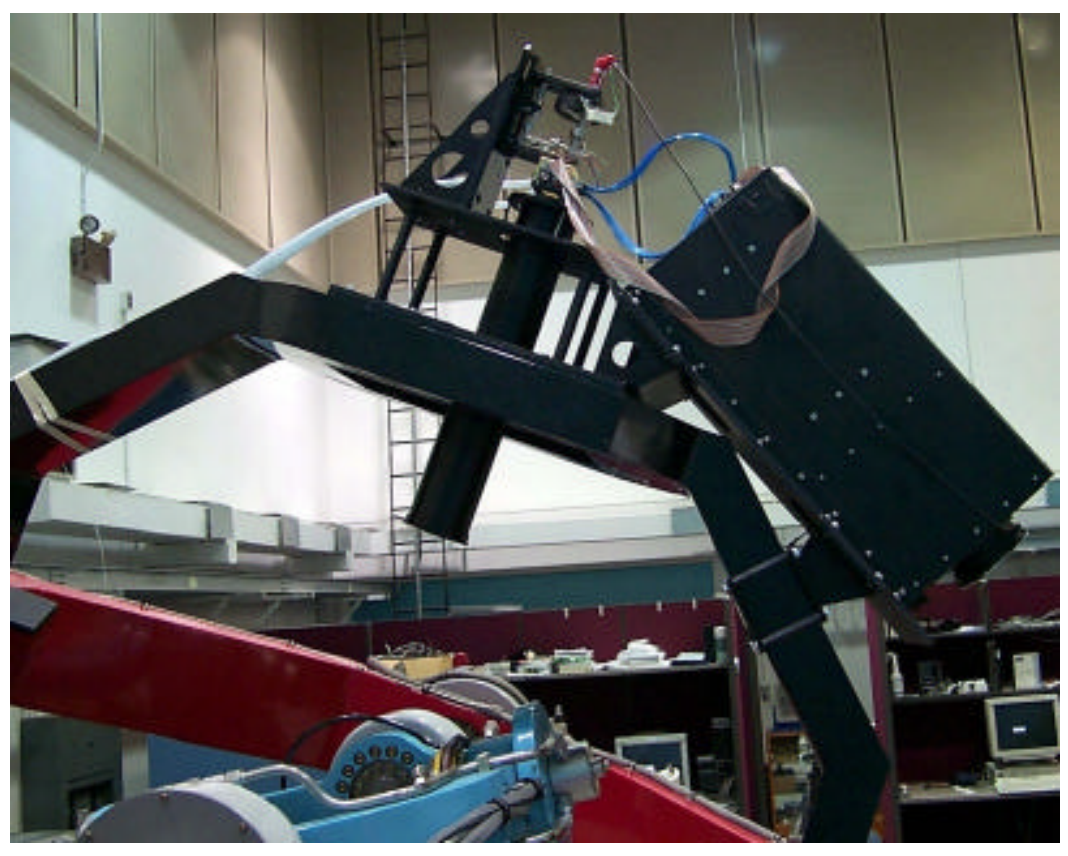

Figure 7 - FMS Mounting of HTC Projector 


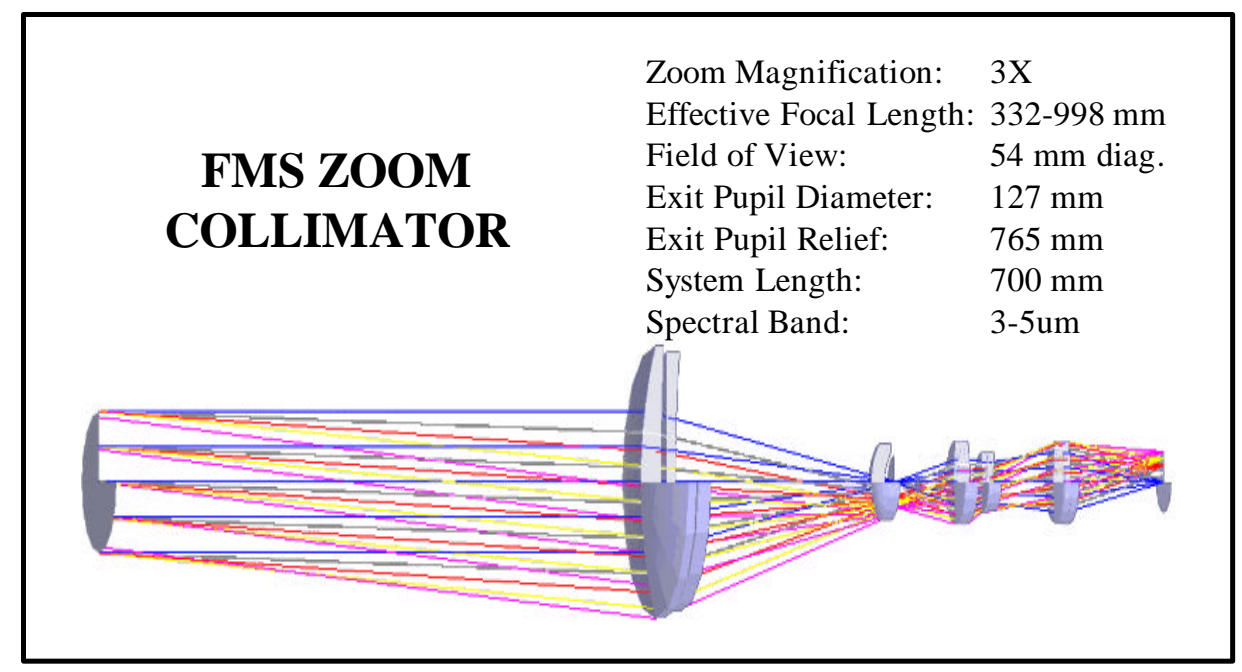

Figure 8 - Zoom Collimator for FMS HWIL Testing

\subsubsection{Extended Analog Cabling for FMS Mounting of the HTC Projector}

Currently the major limitation to the integration and application of the Honeywell emitter array based IR scene projectors into the 5-axis FMS setup is the considerable mass associated with the analog drive electronics for this projector system. The "analog interface electronics" (AIE) currently weigh $\sim 70 \mathrm{lbs}$ and occupy a volume of $\sim 3 \mathrm{ftx} 1.5 \mathrm{ftx} 1 \mathrm{ft}$. An original requirement to keep the interface cables between the AIE and emitter as short as possible typically limits their separation to less than 3 feet. AMCOM RDEC is investigating the performance tradeoffs associated with extending the length of this interface cable to allow for the removal of the AIE from the outer axis of the FMS. This configuration would return much of the capability to the outer axis lost by the addition of the cumbersome electronics package.

Noise increases associated with the additional cable length ( 16ft) have been measured and appear to be on the order of the least significant bit of the 14 bit input. Computer Science and Applications (CSA), manufacturer of the electronics, has also performed noise measurements on an extended length cable and reached similar conclusions. This additional noise associated with the use of this cable is minimal and should not adversely impact most HWIL tests. Figure 9 shows the IRSP as configured on the FMS using the extended length cable to remotely locate the AIE. Figure 10 provides a representative plot of the noise measurements associated with a standard length $(\sim 2 \mathrm{ft})$ and extended length cable. The light (yellow) plot in each is the electronics output as measured off of a 'low-noise' test lead. The darker (green) plots represent the short cable output (upper) and long cable output (lower). The commanded level shown is an input equal to the $13^{\text {th }}$ bit. Oddly, the shorter cable appears noisier than the longer cable. This anomaly, along with the ringing, is probably an artifact of the test setup. A refinement of the noise measurement is planned in the near future.

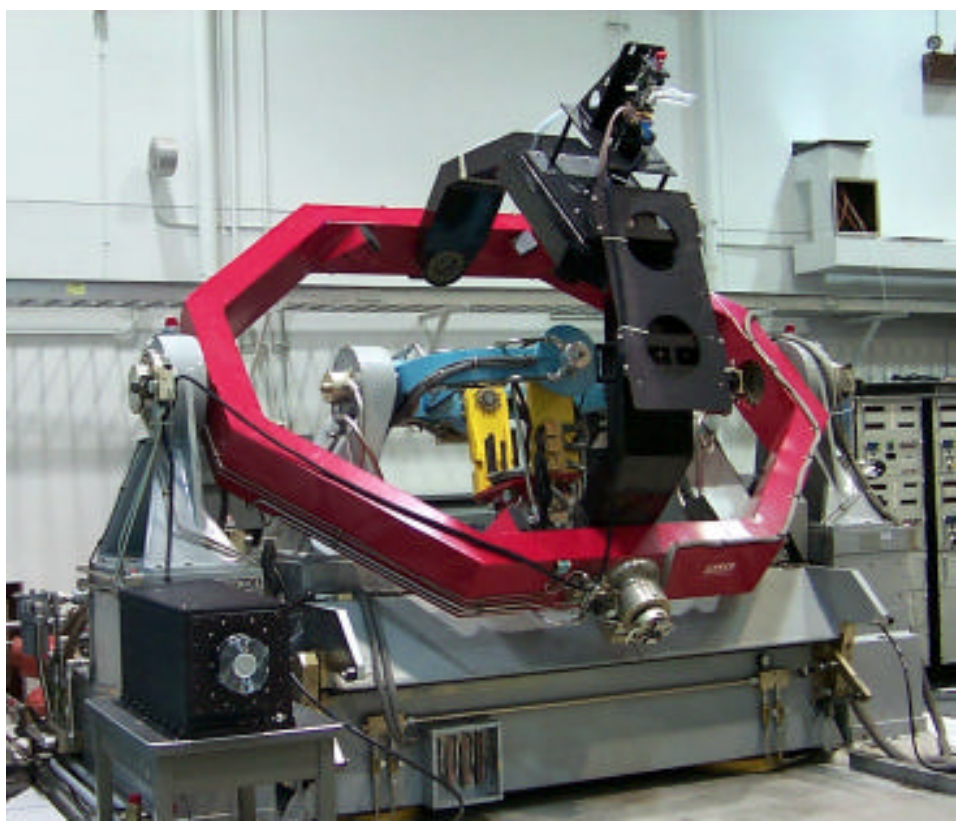

Figure 9 - Extended AIE Cable FMS Mounting 


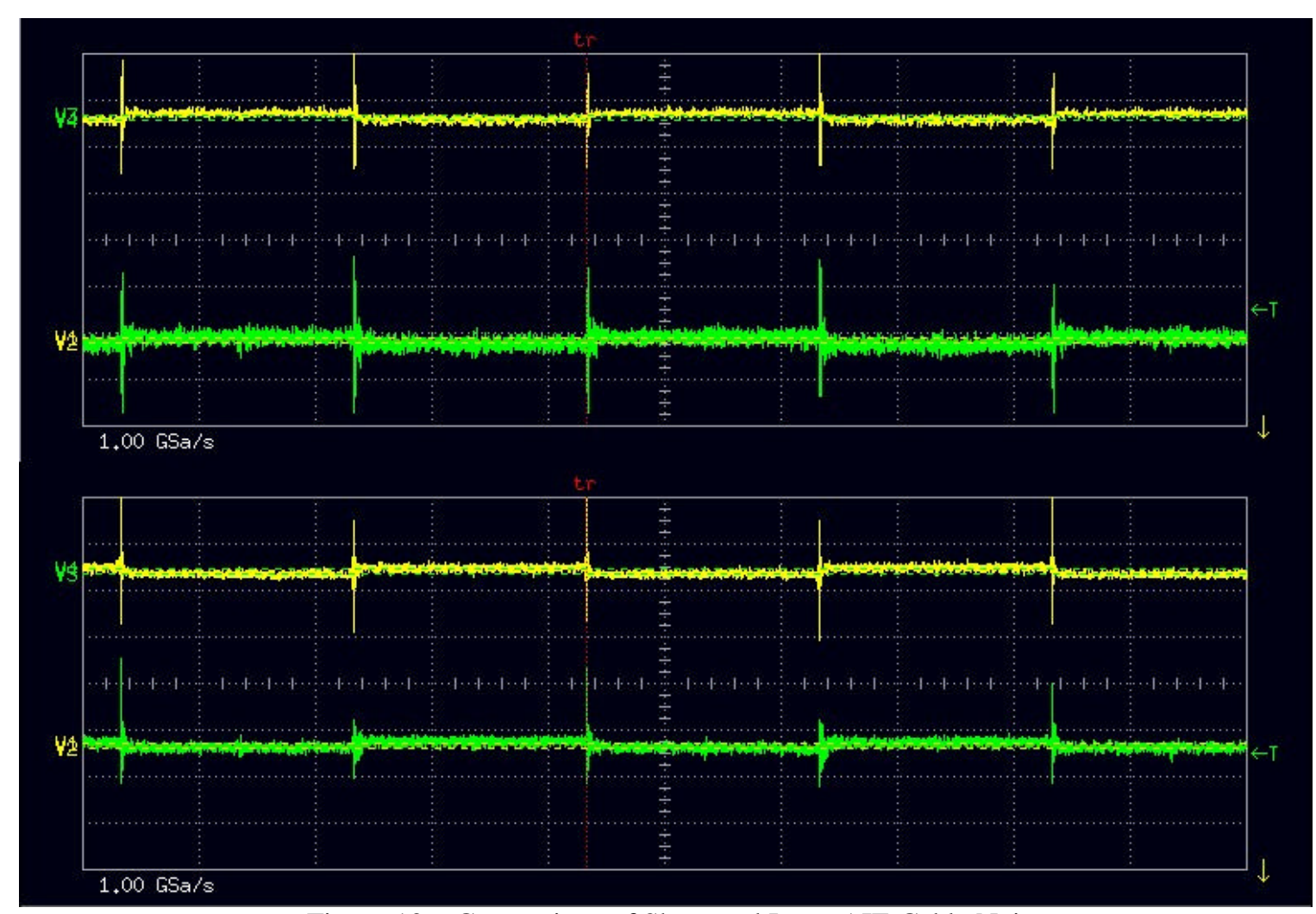

Figure 10 - Comparison of Short and Long AIE Cable Noise

\subsubsection{Near-term Additions}

\subsubsection{MSSP Emitter Array}

Honeywell Corporation, in their ongoing development of new emitter array types, have developed their latest generation emitter arrays under the designation "Multi-Spectral Scene Projector" (MSSP). These arrays use a thinner "Gen3" emitter and support snapshot updating. Through a tri-service program entitled the 'Central Test and Evaluation Investment Program' (CTEIP), AMCOM will shortly take delivery of several of these MSSP arrays. Near-term plans include the characterization of the emitters response as well as verification of the snapshot mode of operation.

\subsubsection{Snapshot Drive Electronics}

The operation of the MSSP array in the "snapshot" updating mode requires a modified set of drive electronics. AMCOM will shortly take delivery of an additional set of these drive electronics capable of operating the MSSP arrays in the "snapshot" mode.

\subsection{MULTI-SPECTRAL INFRARED ANIMATION GENERATION EQUIPMENT (MIRAGE)}

In its efforts to remain at the forefront of HWIL testing, AMCOM RDEC has recently acquired the latest emitter-array based scene projector system. This new emitter technology has been developed through a partnership between Santa Barbara Infrared, Indigo Systems, and the Rockwell Technology Center. Like the current Honeywell emitters, the MIRAGE consists of 512x512 thermal emitters located within a small vacuum chamber on a thermal heatsink. The full IRSP consists of this emitter 'engine' and the thermal control and command electronics sub-systems. Details on the specifications of this system may be found in other papers within this proceedings ${ }^{5}$. 


\subsubsection{Integration}

The MIRAGE system is being evaluated within the AMCOM laboratories to determine the test envelope in which the system can be successfully applied to HWIL testing. Interface checkout and testing of the major sub-system assemblies is being performed. Additionally, AMCOM is developing a custom system control software (SCS) package for the MIRAGE projector based upon the GUI developed for the Honeywell based projectors. System configuration, calibration, operation, and maintenance will be supported through a Windows based GUI. Figure 11 shows the system as configured in one of the AMCOM laboratories.

\subsubsection{Characterization}

Performance characterization of the MIRAGE IRSP is ongoing within the AMCOM RDEC HWIL facilities. Image quality, response time, operability, and dynamic range performance are being investigated in order to characterize this system. One performance parameter measured to date is the emitter response time. The emitters' $10-90 \%$ rise time was measured to be approximately $17 \mathrm{msec}$.

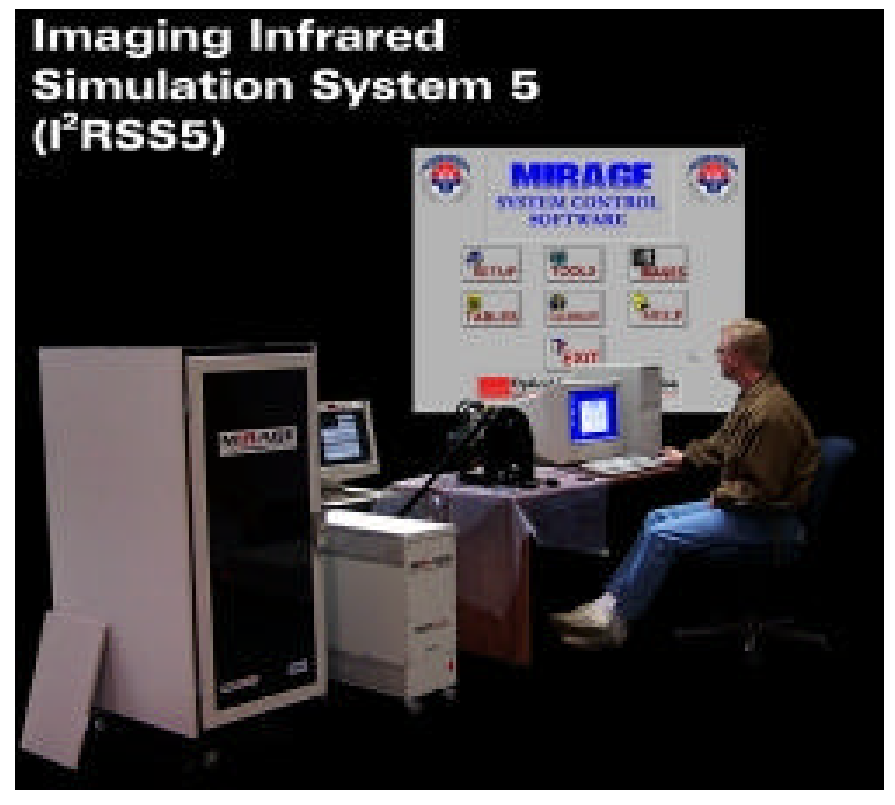

Figure 11 - MIRAGE Projector System

\subsection{DIGITAL MIRROR DEVICE ${ }^{T M}$ BASED IR PROJECTORS}

Optical Sciences Corporation (OSC) is developing a dynamic IR scene projector based upon the Texas Instruments Digital Micromirror Device (DMD ${ }^{\mathrm{TM}}$ ). OSC has successfully demonstrated a prototype DMD IR projector operating in both binary and pulse width modulation (PWM) mode.

\subsubsection{Overview of the DMD Technology for IR Applications}

\subsubsection{DMD Background}

The DMD is a micro-electromechanical system (MEMS) which has a 2-D array of individually controlled aluminum micromirrors. The DMD is the spatial light modulator in TI's Digital Light Processing ${ }^{\mathrm{TM}}$ (DLPTM) system. DLPs are manufactured by TI and sold to OEMs for use in display products such as business projection systems. DMDs are currently commercially available in formats up to $1024 \times 768$.

Each micromirror in the DMD can reflect light in one of two directions $\left( \pm 10^{\circ}\right.$ mechanical) depending upon the state of the underlying memory cell (SRAM). With proper illumination, each mirror will reflect light into the pupil of the optical system when a "1" is written to its SRAM and out of the optical system when a "0" is written to its SRAM. The device is therefore binary in nature. The switching speed on the individual mirrors is approximately $10 \mathrm{usec}$. In commercially available visible projector systems, intensity control is achieved by binary pulse width modulation. The binary image on the array can be updated at a rate of approximately $5000 \mathrm{~Hz}$, and a global reset allows the entire image to be cleared in approximately 20 usec.

\subsubsection{Issues for Application to IR Projection}

The DMD has previously been proposed for dynamic IR scene projection. However, until now there has not been a successfull demonstration of a system. In addition, the IR projector community had significant issues with previously proposed designs. These issues included exit pupil illumination, limited dynamic range, and temporal aliasing/synchronization issues resulting from PWM. The most significant of these issues is the temporal aliasing resulting from the PWM. All of these issues have been addressed in the OSC design. 


\subsubsection{Proof-of-Principle IR DMD Projector with COTS Electronics}

OSC began an internal R\&D effort in December 1996 to investigate the use of the DMD as the basis for a dynamic IR scene projector. A concept for addressing the issues with the DMD discussed above was developed. A demonstration of the DMD in the IR was conducted in June 1998. The DMD was modified for operation in the IR, but the standard electronics were used. A sample image from the demonstration system is shown in Figure 12. Because the electronics were not modified in this system, the temporal aliasing effects due to the PWM can be seen as horizontal bars in the image.

Although this system has flicker, it is very useful for testing certain types of IR sensors. With proper frame syncing, staring sensors which have long integration times, such as $\mathrm{PtSi}$, will not have temporal aliasing due to the PWM.

\subsubsection{Binary IR DMD Projector}

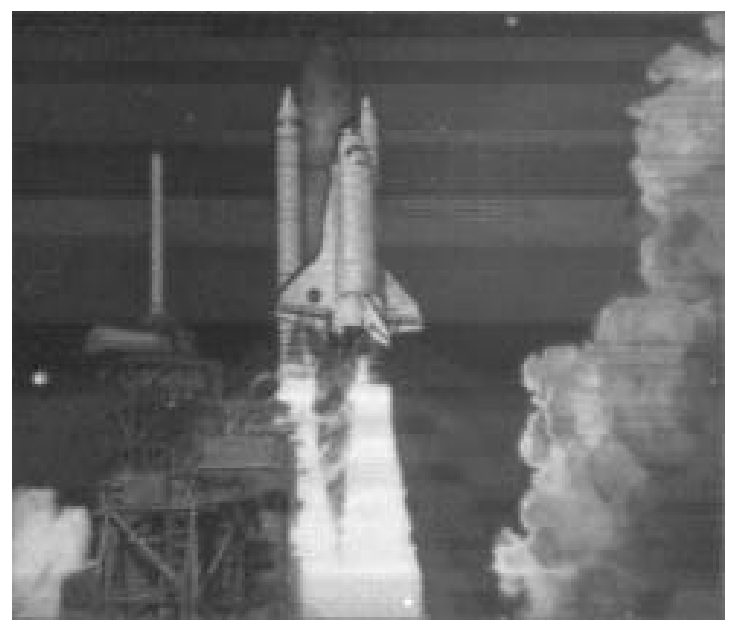

Figure 12 - Sample Image from IR DMD Projector with COTS Electronics

OSC has prototyped a DMD-based IR projector which is capable of operating in two modes: binary and PWM. The first of these configurations is a binary (1-bit) projector capable of projecting high resolution, high-frame rate scenes and test patterns. The projector is virtually flickerless, with only a brief reset occurring each frame to prevent hinge memory in the micromirrors. The projector is compact and can be produced at a low-cost compared to emitter array systems.

\subsubsection{Design of Binary IR DMD Projector}

The projector consists of a single customized DMD, illumination source, collimating optics, and custom drive electronics. The DMD was modified for use in the IR by replacing the standard window with an IR transmissive window. The DMD has a format of $800 \times 600$, but only a $640 \times 480$ region is currently driven by the electronics. A broadband IR source is used for illumination and was designed to ensure that the exit pupil was uniformly illuminated. The system includes an f/3 MWIR collimator, but has also been tested in the LWIR. Custom drive electronics were designed to allow the DMD to operate in binary mode without PWM. The drive electronics are currently capable of controlling a 640x480 region at approximately $5000 \mathrm{~Hz}$. However, the scene generator interface is presently limited to a lower frame rate. The prototype system is in breadboard format, but future form factored systems will be compact, occupying a volume of approximately 10"x6"x6".

\subsubsection{Performance of Binary IR DMD}

Table 2 below summarizes the performance characteristics of the DMD binary projector. An example image collected from the prototype DMD binary projector system is shown in Figure 13.

\begin{tabular}{|c|c|}
\hline Parameter & Performance \\
\hline \hline Spectral Range & Broadband (UV to LWIR) \\
\hline Format & $640 \times 480$ \\
\hline Pixel Pitch & $17 \mathrm{um}$ \\
\hline Maximum Frame Rate & $5000 \mathrm{~Hz}$. \\
\hline Address Mode & Snapshot \\
\hline Max. Duty Factor & $\sim 97 \%$ \\
\hline Amplitude Resolution & 1 bit \\
\hline Dynamic Range & $\sim 16: 1 \mathrm{MWIR}$ \\
& $\sim 1.3: 1 \mathrm{LWIR}$ \\
\hline Apparent Temperature & $25^{\circ} \mathrm{C}-125^{\circ} \mathrm{C}$ MWIR \\
Range & $25^{\circ} \mathrm{C}-45^{\circ} \mathrm{C}$ LWIR \\
\hline Illumination efficiency & $\sim 60 \%$ \\
\hline Pixel Operability & $\mathrm{TBD}(99.99 \%$ goal $)$ \\
\hline Spatial Uniformity & $>98 \%$ \\
\hline
\end{tabular}

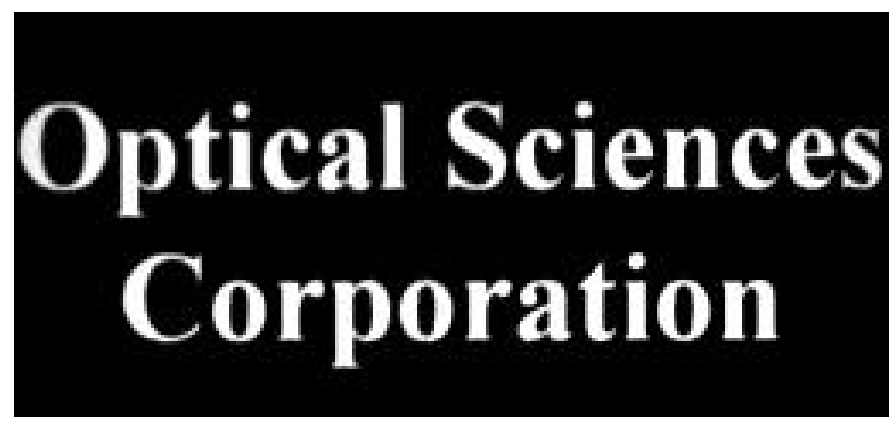

Figure 13 - Sample Image from IR DMD Binary Projector

Table 2 - Summary of IR DMD Binary Projector Performance Parameters 
It should be noted that the apparent temperature range can be increased by increasing the illumination intensity. The dynamic range specification was derived from the ratio of maximum to minimum radiant emittance when the minimum apparent emittance was held near ambient temperature. It should also be noted that DMDs typically have zero dead pixels. Our prototype system has a significant number of dead pixels due to contamination in the assembly process. The process is currently being refined, and it is anticipated that less than $0.01 \%$ dead pixels can be achieved in future systems.

\subsubsection{Pulse-Width Modulation IR DMD Projector}

The second mode of operation for the prototype DMD based IR projector is PWM mode. In this mode, the single-DMD projector can generate various intensity levels using PWM. Even though the projector has flicker, it can be synchronized such that it is not detectable by staring sensors with a nominal integration time. The system can be programmed for varying PWM frequencies to match the integration time of the sensor. The longer the sensor integration time, the more intensity levels that can be achieved. As an example, a typical integration time for an InSb FPA camera is $3 \mathrm{msec}$. With this integration time, the DMD can generate 128 (7-bits) intensity levels. Because of the DMDs binary nature, the intensity levels are very accurate and linear.

\subsubsection{Design of PWM IR DMD Projector}

The design of the system is the same as the binary projector described above except for the control electronics. The control electronics can be programmed to generate any combination of gray scales, frame rate, and PWM frequencies within the capabilities of the DMD.

\subsubsection{Performance of PWM IR DMD Projector}

The performance parameters for the PWM IR DMD are the same as the binary IR DMD except for amplitude resolution, duty factor, and illumination efficiency. Amplitude resolution can be set from 1-12 bits. Duty factor and illumination efficiency may decrease depending upon the PWM parameters chosen.

Two example images collected from the prototype IR DMD projector system operating in PWM mode are shown in Figures 14 and 15. The projector is programmed for 4 bits of amplitude resolution in a $1.5 \mathrm{~ms}$ time period. The image was collected with a $256 \times 256 \mathrm{InSb}$ FPA camera operating at $60 \mathrm{~Hz}$ and set to an integration time of $\sim 1.5 \mathrm{msec}$.

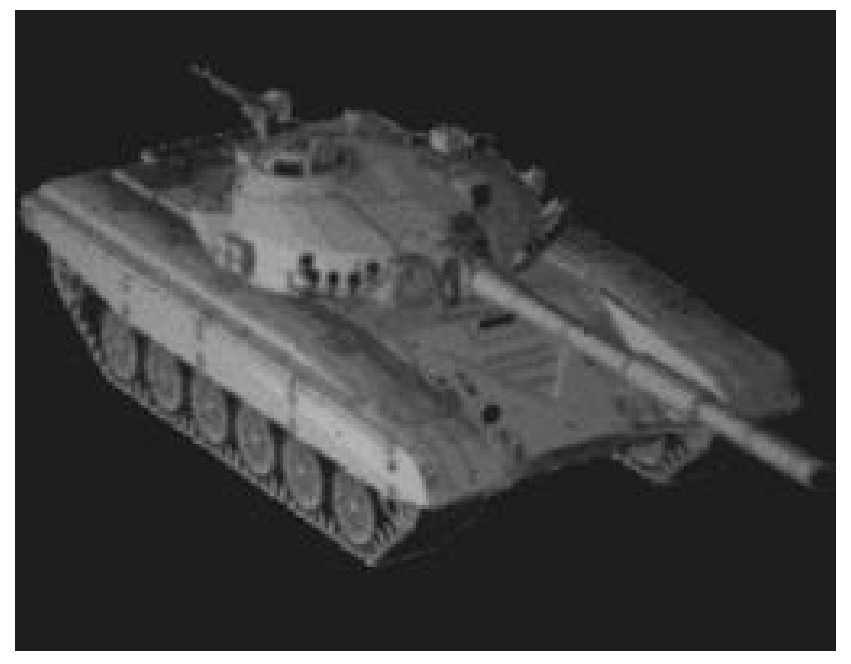

Figure 14: Sample PWM IR DMD Imagery

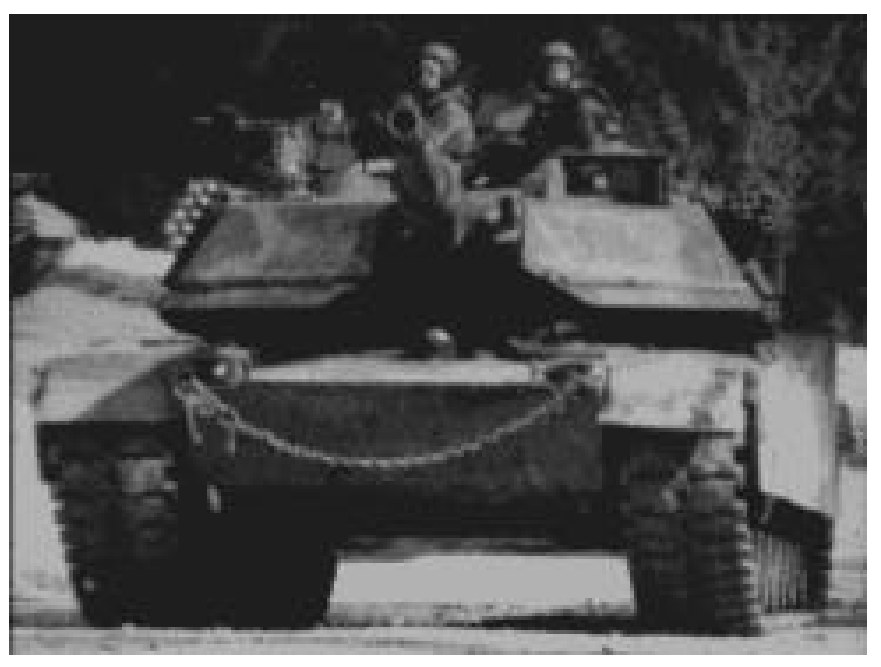

Figure 15: Sample PWM IR DMD Imagery

\subsubsection{Application of IR DMD Projector Systems}

The IR DMD projector can be used to fill the void in IR projector/test systems between static target/test plates and "highend" emitter array/LDAP systems. Advantages of the DMD projector include frame rate, spatial resolution, snapshot update, spatial uniformity, broadband emission, size, and cost. The small size and low mass of the system supports 5-axis FMS mounting and portable test applications. 


\subsection{VISIBLE PROJECTOR SYSTEM}

A dynamic visible projector system has been developed for use in AMCOM's HWIL facilities. The visible projector system will be used for HWIL simulation and testing of seekers which utilize visible band sensors. The visible projector system is based upon the DMD technology, and presently uses standard PWM electronics. The projector has a format of 1024x768, 8bit resolution, and a frame rate of $300 \mathrm{~Hz}$.

\subsection{TRIANGLE TARGET FMS PROJECTOR}

A computer-controlled variable aperture IR projector has been developed by OSC for use on AMCOM's 5-axis flight motion simulator. The projector is designed to simulate high-velocity range closure on triangular-shaped targets such as missile reentry vehicles.

\subsubsection{Design of Triangle Target Projector}

The triangle target projector consist of a motorized target assembly, collimator, and back illumination source. A sketch of the plates is shown in Figure 16. As shown in the figure, the motorized target assembly is designed to move two overlapping plates equally in opposite directions. This allows growth from the centroid of the target such that artificial LOS rates are not introduced into the simulation. The position is accurately controlled using a closed-loop PC based motion controller and an optical encoder for position feedback. The plates are coated with a high emissivity coating and the assembly is back illuminated using an extended area blackbody. Using a blackbody for illumination provides a high emissivity graybody (color-correct) target which supports the testing of dual-band sensors. A photograph of the projector hardware is shown in Figure 17.

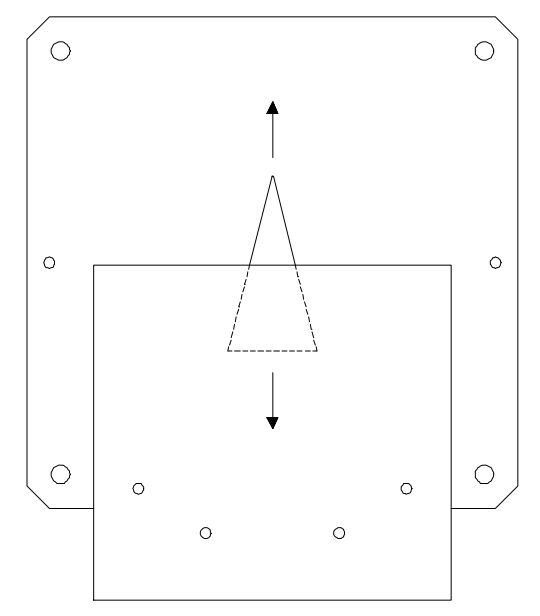

Figure 16 - Sketch of Triangle Target Plates

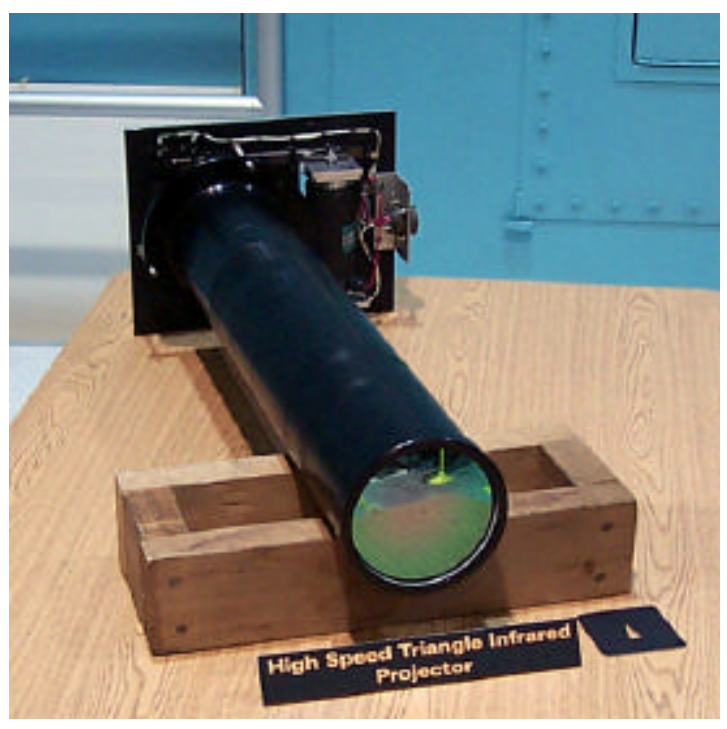

Figure 17 - Triangle Target Projector 


\subsubsection{Performance of Triangle Target Projector}

Table 3 below summarizes the performance characteristics of the triangle target projector. A sample image from the triangle target projector is shown in Figure 18.

\begin{tabular}{|c|c|}
\hline Parameter & Performance \\
\hline Spectral Range & Broadband (2-14 um) \\
\hline Target Emissivity & $\sim 98 \%$ \\
\hline Optics Transmission & $\sim 90 \%$ \\
\hline Update Rate & $100 \mathrm{~Hz}$. \\
\hline Target Size & $0-10 \mathrm{mrad}$ \\
\hline Size Accuracy & $0.2 \%$ \\
\hline Velocity (Target growth) & $0-300 \mathrm{mrad} / \mathrm{sec}$ \\
\hline Acceleration & $0-10 \mathrm{rad} / \mathrm{sec}$ \\
\hline Apparent Temperature Range & $0^{\circ} \mathrm{C}-125^{\circ} \mathrm{C}$ \\
\hline Spatial Uniformity & $>99 \%$ \\
\hline
\end{tabular}

Table 3 -Triangle Target Projector Performance Parameters

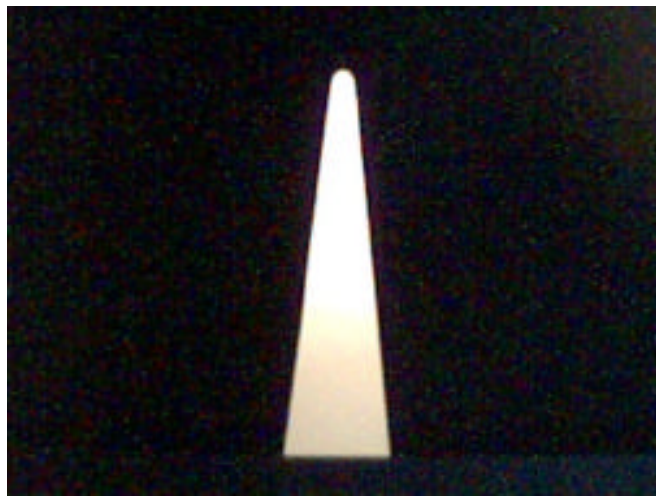

Figure 18 - Sample Image from Triangle Target Projector

\subsection{ACKNOWLEDGMENTS}

This work was sponsored by the US Army Aviation and Missile Command's Research, Development, and Engineering Center under contracts DAAH01-98-C-R090 and DAAH01-99-C-R076. The authors would like to thank Mr. Alex Jolly, Mr. Bill Sholes, Mr. Scottie Mobley, and Mr. Jim Buford, all of USAAMCOM, for their support of our efforts. The authors would also like to thank our colleagues at OSC, Mr. Matt Bender, Mr. Jay Crosby, Mr. Tim Messer, and Mr. Tracy Reynolds, for their support.

\subsection{REFERENCES}

1. J.S. Cole and A.C. Jolly, "Hardware-In-The-Loop Simulation at the U.S. Army Missile Command", Technologies for Synthetic Environments: Hardware-in-the-Loop Testing, Robert L. Murrer, Jr., Editor, Proc. SPIE 2741, pp. 14-19. (1996).

2. J.S. Sanders, R. Roland, D.A. Saylor, D. Cosby, K. Harrison, "Captive flight test-based infrared validation of a hardware-in-the-loop simulation”, Technologies for Synthetic Environments: Hardware-in-the-Loop Testing V, Robert L. Murrer, Jr., Editor, Proc. SPIE 4027 (2000).

3. S.B. Mobley, "Hardware-in-the-loop simulation facility for development, test, and evaluation of multispectral missile systems: update", Technologies for Synthetic Environments: Hardware-in-the-Loop Testing V, Robert L. Murrer, Jr., Editor, Proc. SPIE 4027 (2000).

4. M. Bender, D. Beasley, "Design of a large pupil relief broadband collimator in a MMW/IR HWIL facility", Technologies for Synthetic Environments: Hardware-in-the-Loop Testing V, Robert L. Murrer, Jr., Editor, Proc. SPIE 4027 (2000).

5. S.W. McHugh, J. Oleson, L. Rubin, "MIRAGE: system overview and status", Technologies for Synthetic Environments: Hardwarein-the-Loop Testing V, Robert L. Murrer, Jr., Editor, Proc. SPIE 4027 (2000).

\subsection{TRADEMARKS}

Digital Micromirror Device, DMD, Digital Light Processing, and DLP are registered trademarks of Texas Instruments Incorporated. 